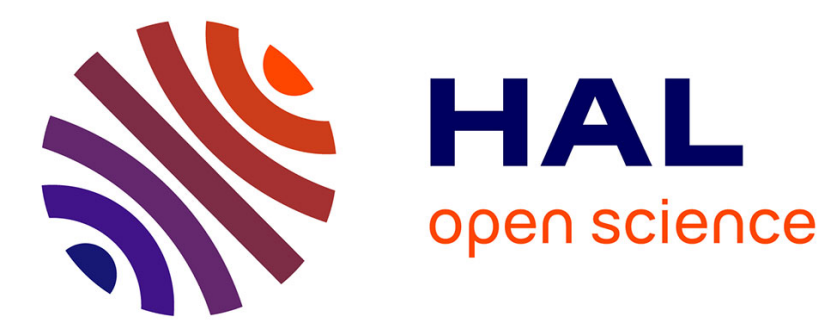

\title{
La communauté francophone de Moscou sous le règne de Catherine II
}

\author{
Vladislav Rjeoutski
}

\section{To cite this version:}

Vladislav Rjeoutski. La communauté francophone de Moscou sous le règne de Catherine II. Revue des études slaves, 1996, 68 (4), pp.445-461. halshs-00292306

\section{HAL Id: halshs-00292306 https://shs.hal.science/halshs-00292306}

Submitted on 1 Jul 2008

HAL is a multi-disciplinary open access archive for the deposit and dissemination of scientific research documents, whether they are published or not. The documents may come from teaching and research institutions in France or abroad, or from public or private research centers.
L'archive ouverte pluridisciplinaire HAL, est destinée au dépôt et à la diffusion de documents scientifiques de niveau recherche, publiés ou non, émanant des établissements d'enseignement et de recherche français ou étrangers, des laboratoires publics ou privés. 
Version abrégée de l'article publié dans la Revue des Etudes Slaves, Paris, LXVIII /4, 1996, p. 445-461.

Vladislav Rjéoutski

\title{
LA COMMUNAUTE FRANCOPHONE DE MOSCOU
}

\author{
SOUS LE REGNE DE CATHERINE II.
}

En 1793, en réponse à l'exécution du Roi de France Louis XVI, Catherine II de Russie signa l'oukase interdisant toutes les relations avec la France.1 Un des points essentiels de cet oukase prévoyait l'expulsion de l'Empire de tous les sujets français qui refuseraient de prêter le serment de n'entretenir aucune relation avec l'étranger. Quel était le nombre de ceux qui étaient concernés par cette mesure en Russie et qui étaient-ils?

Les listes des Français qui prêtèrent ce serment, furent publiées dans le journal SanktPeterbourgskie Vedomosti.2 Les numéros 45-46 du journal contiennent les noms et les prénoms de presque 800 sujets français adultes domiciliés à Saint-Pétersbourg et l'indication des métiers qu'ils exerçaient.3 Environ 900 sujets français et leurs enfants résidaient vers cette époque à Moscou, ce qui ressort des listes publiées dans les numéros 47-51 du même journal pour l'année 1793.4 Dans les listes des Français-Moscovites sont omis, à la différence des listes pour SaintPétersbourg, tous les renseignements concernant les métiers exercés par la communauté de Moscou. En revanche, la ville d'origine des Français-Moscovites est presque toujours indiquée.5 Dans toutes les autres provinces de l'Empire le nombre de sujets français ne dépasse pas 300-400

\footnotetext{
Je saisis cette occasion pour exprimer ma gratitude à M.Wladimir Berelowitch et Mme Jutta Scherrer pour l'aide et le soutien qu'ils m'ont accordé au cours de mes études à l'Ecole des Hautes Etudes en Sciences Sociales à Paris en 1995-1996.
}

Toutes les citations sont reproduites avec l'orthographe de l'original.

1L'oukase du 8 février 1793, « О прекращении сообщения с Франциею, по случаю произошедшего в оной возмущения и умервщления Короля Людовика XVI, и высылке Французов из России, исключая тех, которые под присягою отрекутся от революционных правил, во Франции распространившихся» // Полное собрание законов Российской империи, SPb., 1830, vol. 23, nº 17101.

2Санкт-Петербургские ведомости, 1793, $\mathrm{n}^{\circ} 45-53$.

3Sankt-Peterburgskie vedomosti signalent $\left(1793, \mathrm{n}^{\circ} 46, \mathrm{p}\right.$. 1045) que le nombre de sujets français qui prêtèrent le serment à Saint-Pétersbourg était égal à 786 (bien que les listes en contiennent au plus 765). En y ajoutant les 12 personnes qui prêtèrent le serment plus tardivement (ibid.), on aura le chiffre de 798 où cependant sont exceptés les enfants.

4Ces listes reproduites dans ces numéros (Sankt-Peterburgskie vedomosti, 1793, $\mathrm{n}^{\circ}$ 47-51) contiennent, d'après mes calculs, 906 personnes, y compris les enfants. Les listes sont précédées par les mots suivants: «Список оставшимся в России и по имянному Ея Императорскаго Величества указу, состоявшемуся сего 1793 года Февраля в 8й день, бывшим у присяги, Французам и Франиуженкам находящимся как в столичном городе Москве, так и других городах». Or à juger d'après les listes elles-mêmes, il ne s'agit que des habitants de Moscou.

5 Il est à remarquer que les listes incluent aussi les émigrés francophones des villes comme Bruxelles, Genève ou Luxembourg, bien que le nombre de ceux-ci fût minime. Les originaires francophones de la Belgique, de la Suisse etc. faisaient toujours partie de la communauté française de Moscou, abstraction faite du statut de ses pays. On trouve même dans les listes de la communauté dressées par le vice-consulat de France à Moscou un Espagnol; la raison en est certainement dans la langue (et la culture) française qui étaient un dénominateur commun pour tous ces émigrés. C'est la raison qui nous fait parler de la communauté «francophone ». 
personnes $^{6}$. Bien sûr le nombre absolu de Français en Russie était plus considérable car les cas de la naturalisation n'étaient plus si rares à cette époque. En comptant aussi les enfants, le nombre absolu de Français résidant à cette époque en Russie pourrait être estimé à au moins 2500 personnes.7 A Moscou (avec sa banlieue), en 1793, les Français étaient près de 1000 personnes. Comment une telle communauté s'est-elle formée?

\section{LA NAISSANCE DE LA COMMUNAUTE FRANCOPHONE DE MOSCOU.}

En 1759, le vice-consulat de France s'ouvrit à Moscou. Le premier vice-consul s'appelait Pierre Martin. Il resta à ce poste au moins vingt ans, tout en assurant les fonctions de précepteur dans la famille des princes Tcherkasski.8 Il fut un témoin privilégié de la naissance de la colonie francophone de Moscou.9 Il est naturel que, pendant cette période, il connût parfaitement une grande partie de ses compatriotes moscovites. Cette qualité le distinguait de ses collègues de hauts rangs de Saint-Pétersbourg.10 C'est la raison qui a notamment déterminé un certain degré de confiance (v. ci-dessous) qu'on serait prêt à accorder aux documents rédigés par Pierre Martin, confiance qui est tantôt limitée, tantôt affermie par la comparaison avec d'autres sources d'une fiabilité plus ou moins certaine.11 Parmi les documents qu'on tient de Pierre Martin, deux attirent particulièrement l'attention. Il s'agit de deux listes de la communauté française (ou francophone).

La première est intitulée «Etat des françois, sujets du roi, de leur femmes et enfant actuellement à Moscou et aux environs, au premier janvier mil sept cent soixante deux », et se rapporte à l'année 1762.12 La deuxième était dressée par le vice-consul en 1777 sous le titre «Etat de La Colonie françoise à Moscou et dans cette partie de l'Empire de Russie, pour être mis sous les yeux de Monseigneur de Sartine Ministre et Secrétaire d'Etat ayant Le département de la marine, par le Sr. martin viceconsul à Moscou, au commencement de 1777 ».13 L'établissement de ces listes faisait partie des devoirs de Pierre Martin en tant que vice-consul. Dans l'instruction qu'il reçut de Paris par l'intermédiaire de l'ambassade de Saint-Pétersbourg, au tout début de l'exercice de ses fonctions, il lui fut notifié que :

\footnotetext{
${ }^{6}$ Sur cette question, consulter l'article paru après la sortie de ce texte : Dmitrij A. Rostislavlev, Svetlana L. Turilova, « Francuzy v Rossii v 1793 godu », Cahiers du monde russe, 39(3), 1998, p. 297-320.

7OOn en connaît plusieurs, mais il semble extrêmement important de savoir les chiffres exacts qui sont des indicateurs des processus (l'assimilation, l'influence des facteurs d'ordre politique, économique etc., l'image de la Russie et ainsi de suite) dans le milieu des étrangers en Russie.

8Archives diplomatiques de Paris, Correspondance politique, Russie, vol.100 (année 1777), p.113verso.

9Avant l'immigration entreprise sous le règne de Catherine II, le peu de Français de Moscou étaient pour la plupart d'extraction huguenotte. Jurgen Kämmerer conclut dans son livre Russland und die Hugenotten im 18. Jahrhundert (1689-1789), Wiesbaden, 1978, p.34: «Zur Bildung einer franzosisch-reformierten Gemeinde ist es jedoch in Moskau nicht gekommen. Zu gering war die Anzahl hugenottischer Familien in der alten Hauptstadt ». Il semble que les quelques artisans français arrivés à Moscou aux années 1720 via Saint-Pétersbourg ne s'y éternisèrent pas non plus. V. sur ce sujet les lettres inédites du vice-consul de France à Saint-Pétersbourg (sous le règne de Pierre $\mathrm{I}^{\mathrm{er}}$ ) Henry Lavie, Archives Nationales, Affaires étrangères, B-I-983, f.1-2 etc.
}

10L'attitude des hauts fonctionnaire de la diplomatie française en Russie envers leurs compatriotes expatriés et la bonne ou mauvaise connaissance avec leur vie qui est fonction de cette attitude fait l'objet d'une étude à part et nous n'en ferons pas de résumé ici.

11P.ex. N. A. Najdenov, Материаль для истории московского купечества, М., 1883-1889, t. 1-9.

$12 \mathrm{~J}$ 'exprime ma reconnaissance à Vladimir Somov qui m'a indiqué ce document. ADN, Moscou, vice-consulat, $\mathrm{n}^{\circ}$ 1, p. 99-101.

13Archives Nationales de France (AN), Affaires étrangères, B-I-480, f. 213-223. 
Il sera très bien aussi qu'il fasse à son loisir un état général de tous les françois établis tant à Moscou qu'aux environs, en notant par autant de colonnes ce qu'il apprendra du tems de leur arrivée, de leur âge, de leur état de marié ou non marié, de leurs enfans, de leurs talens, métiers ou fonctions et surtout de leurs bonnes ou mauvaises qualités, mais il doit plutôt recueillir ces connaissances que d'en faire des recherches avec affectations, puisqu'elles doivent être secrettes.14

La liste dressée en 1762 reproduit le nom et le prénom des Français résidant à Moscou cette année-là, fait mention de leurs femme et enfants, donne parfois quelques indications, toujours succinctes, sur l'origine de l'immigré et le métier qu'il exerce. Ces informations sont complétées par d'autres qu'on peut tirer des autres documents du même registre dont la liste susmentionnée fait partie: procurations, légalisations des extraits de baptême, de décès ou de mariage, actes de résolution, assignations, etc. La liste de 1777 est de beaucoup plus régulière. Elle donne le nom, souvent le prénom des hommes; indique s'ils sont mariés, veufs ou célibataires; combien d'enfants ils ont; de quelle région ou ville de France ils sont originaires (pour à peu près 140 familles et individus, soit 283 personnes, sur le total d'environ 210 familles et individus); enfin quels métiers ils exercent (pour 89 personnes adultes sur le nombre total d'à peu près 270 adultes mentionnés dans la liste).

Dans la liste dressée en 1762 sont mentionnées seulement 156 personnes, hommes, femmes et enfants. Même si l'on y ajoute quelques autres Français qui résidaient à Moscou à cette époque selon le registre du vice-consulat dont on dispose et qui couvre les années 1759-1764, leur nombre absolu à Moscou au début des années 1760 n'excédait apparemment 200 personnes, femmes et enfants compris, ce qui n'est qu'une petite poignée en comparaison avec la colonie des années 1770. La liste de 1777 comporte environ 650 personnes, hommes, femmes et enfants.15 En 1762 les Français ne sont pas encore concentrés dans un quartier, plusieurs vivent dans les hôtels de la noblesse. Ainsi, un Jean Mathieu Chauvin, «précepteur des enfants d'Alexandre Petrowitch Akinfioff » ou une Marguerite Deschamps demeurant on ne sait en quelle qualité chez le prince Alexis Sergejevitch Golitsyne. C'est aussi le cas d'une Marie Magdeleine Françoise de Surville logée chez le prince Nikita Iourievitch Trubeckoï. Probablement quelques uns résident dans le faubourg des Etrangers (Nemetskaïa sloboda), mais N. Martynova-Poniatovskaïa qui a pris le soin d'étudier les listes des premiers recensements (revizskie skazki) n'y en a relevé presque aucun.16 Les Français catholiques et leurs compatriotes protestants étaient les uns comme les autres en minorité dans les paroisses à prédominance polonaise et allemande. De quoi on conclut provisoirement que les Français de Moscou des années 1750 - début des années 1760 ne formaient pas encore de communauté vraiment organisée ni à plus forte raison structurée.

La constitution de la colonie francophone de Moscou coïncide donc avec l'époque du règne de Catherine II. Mais tout en étant le fruit des initiatives de la grande souveraine, la communauté de Moscou se forma plutôt malgré les projets de l'immigration conçus et réalisés sous Catherine II, que grâce à eux.

14 «Instruction Pour Le Sr. Pierre Martin Etabli Vice-consul de france à Moscou et dépendances, par patentes du Sr. De Cury de St. Sauveur Consul général de france En Russie en date Du premier avril 1760 », ADN, Moscou, vice-consulat, $\mathrm{n}^{\circ}$ 1, p. 23-30.

$15 \mathrm{Il}$ est impossible d'évaluer, d'après cette liste, quel était le nombre exact de Français à Moscou à cette époque. D'abord parce que, dressée par une personne qui avait pour l'execution de cette tâche des moyens quand même très limités, cette source n'est bien sûr pas exhaustive ni libre de quelques erreurs. Et puis, parce que le nombre d'enfants dans plusieurs familles n'est pas précisé.

16N. G. Martynova-Ponjatovskaja, Материалье к истории франиузской книжной торговли в Москве // Сборник статей Публичной библиотеки имени Ленина, М., 1928, vol. 1, p. 113 sqq. 
Il est notoire que l'immigration organisée par Catherine II était pensée comme rurale et non urbaine, à la différence de l'immigration du temps de Pierre $\mathrm{I}^{\mathrm{er}} .17$ Une autre particularité de la politique migratoire de cette époque, bien différente aussi sur ce point de la politique de Pierre le Grand, était une certaine confusion des critères selon lesquels les immigrés étaient choisis et un laisser-aller général en ce qui concernait la qualité de l'immigration au profit de la quantité. Le caractère rural de la colonisation d'une part et les caractéristiques professionnelles de beaucoup d'immigrés en désaccord flagrant avec la direction de la politique migratoire d'autre part, sont à compter parmi les causes de la naissance de la communauté francophone de Moscou.

Les immigrés étaient invités à s'établir principalement sur les territoires vides, surtout sur ceux qui étaient recommandés par le gouvernement. Par contre, la fixation dans les villes, sans être vraiment interdite, était somme toute mal vue. Pourtant, beaucoup d'immigrés d'origine française n'ont pas manqué cette occasion, bien que ceux qui choisissaient à se fixer dans les capitales ne fussent exempts des impôts et toutes sortes de charges que pour une période de cinq ans et non de trente comme les colons à Saratov. En 1777, comme on vient de le signaler, il y avait non moins de 650 Français à Moscou. Seuls deux ou trois reçurent l'aide du gouvernement pour l'établissement des entreprises, notamment Fazy et Ferrier pour la fondation d'une fabrique de montres à Moscou.18 Les autres vinrent probablement à leurs propres frais ou prirent la fuite durant le trajet de Saint-Pétersbourg à la région de la Volga.19 Mais pour un grand nombre le chemin à Moscou dura plusieurs années.

Parmi les colonies fondées par les immigrés dans la région de la Volga il en y avait une qui s'appelait Rosochi. C'était la seule où l'élément français était dominant. En outre, à Saratov même s'établirent quantité de Français, de Suisses et de Wallons.20 En 1775, une commission spéciale était chargée de trier tous les colons d'après leur aptitude à s'occuper de l'agriculture. En résultat 529 furent reconnus incapables de cultiver la terre, reçurent les passeports leur permettant de choisir un autre mode de vie en changeant le lieu de domicile. Une grande partie de ces derniers se retrouvèrent sous peu dans les capitales.21 Pierre Martin écrivait dans le document cité qui se rapporte à l'année 1777 :

La grande colonie françoise était à Saratoff, mais elle a été presque toute dispersée par les ordres de L'impératrice, qui a permis aux familles qui La composaient de se répandre dans L'Empire pour y faire usage de leurs talents et y chercher à vivre. Il est cependant resté plusieurs de ces familles à Saratoff, ainsi que dans d'autres colonies mélées de toutes les nations.22

Or d'après la liste de Pierre Martin de 1777, environ 150 Français, hommes, femmes et enfants, domiciliés à Moscou en 1777 étaient peu avant des habitants de Saratov et de sa province.

Le sens profond et la place de cet événement dans l'histoire de l'immigration française en Russie sont clarifiés par un document intitulé Observations Sur La Levée des Colonies Russes et

17Cf. les opinions sur ce sujet: R.Bartlett, Human Capital. The Settlement of Foreigners in Russia, 1762-1804, Cambridge University Press, Cambridge, London, New York, New Rochelle, Melbourne, Sydney, 1979, p.53 sqq. et A. A. Kizevetter, Отзыв о сочинении Г. Г. Писаревского Из истории иностранной колонизации в России 8 XVIII веке // Отчет о 52-м присуждении наград гр. Уварова, Записки Императорской Академии наук по историко-филологическому отделению, vol. XI, nº 3, SPb., 1912, p. 151-200 et en particulier p. 159.

18R. Bartlett, op.cit., p. 168-169, 308.

19Cf. G. G. Pisarevskij, Хозяйство и форма землевладения в колониях Поволжья в ХVIII и в первой четверти XIX в., Rostov-sur-le-Don, 1916, p. 23.

20V. F. Šišmarev, Романские поселения на юге России // Труды Архива АН СССР, vol. 26, L., 1975, p. 111 sqq.

21Pisarevskij, op.cit., p. 36; Bartlett, op.cit., p. 106.

22AN, Affaires étrangères, B-I-480, p. 218. 
L'Emigration des Familles Françoises provenant de la partie des Archives de la Bastille qui est conservée à la Bibliothèque nationale de Russie.23 Il en découle que nombre de Français étaient recrutés hors de France, dans les communautés francophones de l'émigration, dans les villes comme Hambourg ou Francfort. Différentes étaient les voies qui les y avaient amenés : les uns avaient émigré lors des campagnes de la colonisation en Prusse : d'autres avaient déserté de l'armée française : d'autres encore, les huguenots, avaient fui les persécutions religieuses... Pierre Martin signale la présence d'au moins 18 déserteurs (sur 48 hommes adultes) parmi les Français venus de Saratov à Moscou.24 L'absolue majorité des Français engagés en Allemagne étaient des habitants urbains. Ils l'étaient souvent encore avant de s'expatrier et de s'établir dans les villes allemandes. Il n'y avait parmi ceux-là que très peu de gens qui étaient liés à l'agriculture, si ce n'est les quelques anciens vignerons établis à cette époque à Francfort. Les immigrés français «levés » dans ces villes maîtrisaient en général des professions urbaines.25 A l'arrivée dans la région de la Volga, beaucoup étaient mis devant un choix des plus difficiles, surtout si on prend en compte l'insistance de la Chancellerie de tutelle des colons étrangers (Kantseliariia opekounstva inostrannykh26) qui forçait les colons de changer leur métier pour celui de laboureur. La fuite dans les capitales était donc une conséquence logique du fait que beaucoup de colons français se montrèrent réfractaires, voire incapables à s'adapter aux conditions qu'ils n'avaient pas choisies. La fuite devint une véritable débandade quand les colons comprirent que l'enseignement de leur langue était un excellent gagne-pain en Russie.27 L'effritement de l'élément français dans la région de la Volga était de surcroît accéléré par des calamités qui poursuivaient les colonies depuis le début de la colonisation: les mauvaises récoltes se succédaient, beaucoup de colonies étaient déficitaires au cours de dix premières années de leur existence, voire plus; en 1774, Saratov fut beaucoup ravagé par un terrible incendie et sa dévastation était achevée par les troupes de Pougatchev trois mois plus tard.28 Et ce n'est pas un hasard si Pierre Martin parle en pleine connaissance de cause des événements qui eurent lieu à une distance énorme de Moscou: «Il ne reste plus guère que quatre ou cinq mille de ces familles, Le reste étant péri de misère et de maladies, ou massacrés par Pougatchoff, ou emmenés en esclavage par les tartares $\gg .29$

Aussi, un des facteurs importants pour la constitution de la communauté francophone de Moscou doit-il être cherché dans la contradiction entre le caractère principalement urbain des immigrants

23Département des manuscrits de la Bibliothèque nationale de Russie (BNR, Saint-Pétersbourg), Archives de la Bastille, autographe 121, n 107. Très abîmé, le document ne fut que mentionné par Paul d'Estrée, Une colonie franco-russe au XVIII siècle, Revue des Revues, 1896, §19. Il fut reproduit en entier pour la première fois (si on exclut la publication dans le catalogue tapuscrit interne de la Bibliothèque « Документьл из Бастильского архива: аннотированный каталог», L., 1960, par A. Ljublinskaja) par Roger Bartlett, op.cit.., p. 250-255.

24AN, Affaires étrangères, B-I-480, p.213-223.

25Ainsi, on y rencontre des fabricants, officiers et bas-officiers levés en Hollande, des « Déserteurs, fabricants de bas, forgerons reformées pour la plus part » engagés à Wurms, «20 familles françaises prés composées de Cloutiers, Tisserans, Bucherons tirées des Ardennes » embauchées à Furth etc., R.Bartlett, op.cit., pp.250-253. Cf. à ce propos l'opinion de Šišmarev, op.cit., p. 113.

260rganisme qui avait existé de 1763 à 1782 et était chargé de la direction du recrutement et de l'établissement des colons. Le fonds de la Chancellerie aux Archives d'Etat des actes anciens (RGADA) à Moscou (F. 283, 210 dossiers) contient les documents concernant le recrutement des colons dans les pays d'Europe, leur établissement dans les différents gouvernements de l'Empire, y compris dans la province de Moscou, les listes de colons (17681769), etc.

27Cf. Pisarevskij, op.cit., p. 6-10.

28Cf. ibid.; Труды Нижне-Волжского областного научного общества краеведения, vol. 35, t. 2, recueil historique, Saratov, 1928; V. V. Mavrodin, Об участии колонистов Поволжья в восстанииии Пугачева // Крестьянство и классовая борьба в феодальной России: сборник статей памяти И. И. Смирнова = Труды института историиии, Лениградское отделение, АН СССР, vol. 9, p. 400-412.

29AN, Affaires étrangères, B-I-480, p. 218. 
français des années 1760 et la tendance générale de la politique migratoire du gouvernement russe à cette époque. Une autre raison était l'intérêt toujours croissant parmi la noblesse russe pour tout ce qui était lié à la France, à commencer par la langue française. 30 Ainsi, les membres de la famille la plus nombreuse parmi celles arrivées de la région de Saratov à Moscou, les Dellesalle, gagnaient leur vie en enseignant le français dans les pensions qu'ils avaient fondées et ce métier s'était transmis à nombre de descendants des premiers Dellesalle venus en Russie.31 Plus tard, un des mécanismes essentiels de l'agrandissement et du renouvellement de la communauté était une migration des Français appelés par leurs conjoints, parents, anciens voisins à venir à Moscou, phénomène qui a reçu le nom de «chain immigration » dans la littérature anglo-américaine. C'est le mécanisme qui est la cause du flux important des immigrants de quelques petites villes de France en Russie.32

En 1777, environ la moitié de tous les Français de Moscou étaient originaires de quatre villes ou régions de France, de Lorraine (en tête avec 13\% des Français de la colonie), de Paris, du Dauphiné, de Lyon (10\%).33 Il a déjà été dit que nombre d'immigrés arrivèrent en Russie via les villes allemandes. Seize ans après, en 1793, parmi les sujets français à Moscou, 20\%, c'est-à-dire le groupe le plus nombreux, étaient nés en Russie de parents français. Leur emboîtaient le pas d'anciens Parisiens (16\%), des originaires de l'Alsace et de la Lorraine (presque 15\%), tandis que les Lyonnais étaient bien moins nombreux qu'en 1777 (moins de 3\%).34 On voit ainsi que durant la deuxième partie du règne de Catherine II quelques changements notables sont intervenus dans la constitution de la communauté de Moscou. D'abord, c'est l'apparition des deuxième et troisième générations des immigrés français à Moscou, ce qui témoigne d'un important taux de sédentarisation de l'immigration française dans cette ville. Ensuite, une diminution notable du flux des immigrés de Lyon qui s'explique probablement par le piètre succès de la production de soie à Moscou. Enfin, une augmentation du nombre d'immigrés d'Alsace. Les mêmes tendances se profilent à Saint-Pétersbourg au début du XIX ${ }^{\mathrm{e}}$ siècle. $^{35}$

30Même dans la région de la Volga, pourtant extrêmement pauvre en ressources humaines, les colons français connurent un succès considérable en devenant précepteurs. Cf. Pisarevskij, op.cit., p. 7.

31V. p.ex. M. Del'sal', Краткая историческая записка о пансионе М. Дельсаль, 1869, n 1, p. 35-38.

32P.ex. des originaires de Lunéville. En 1793 on compte au moins 24 anciens Lunévillois à Moscou, SanktPeterburgskie vedomosti, 1793, $\mathrm{n}^{\circ}$ 47-51. En 1819 un notaire originaire de Lunéville nommé Nicolas Touvenel conclut à Saint-Pétersbourg le mariage avec Ursule Socerot, fille du dentiste à la cour de Russie Joseph Socerot, lui aussi originaire de Lunéville, bien que sa fille fût née déjà à Saint-Pétersbourg. Archives diplomatiques de Nantes, Saint-Pétersbourg, consulat, état-civil, $n^{\circ} 1$, Registre des naissances, mariages et décès. An X à 1821, p. 42. On peut penser que ce n'était pas un cas unique.

33D'après la liste de 1777, AN, Affaires étrangères, B-I-480, p. 213-223. V. Šišmarev, sur la base de plusieurs sources approximatives a conclu que la quantité de véritables Français ne dépassait dans la région de la Volga 70 personnes. Sur ce nombre, la plus grande partie étaient originaires du Nord-Est et de l'Est: Ile-de France, Orléan, Berry, Picardie, Artois, Champagne, Lorraine, Bourgogne, Alsace, Franche-Comté. Beaucoup moins sont représentés l'Ouest et le Sud-Ouest. La divergence d'avec le document susmentionné des Archives de la Bastille n'est qu'apparente, pense V. Šišmarev. Beaucoup de « Français » d'Alsace et de Lorraine étaient, selon lui, Allemands par leur langue. (V. Šišmarev, op.cit., p. 109-110). D'autant plus il n’y a pas de contradiction entre ces données et celles qui viennent de l'analyse de la liste de Pierre Martin de 1777: les chiffres qu'on donne se rapportent presque exclusivement à ceux des immigrés français de Moscou qui vinrent en Russie eux-mêmes et ne furent pas engagés par les « recruteurs » (vyzyvateli), peut-être aussi à quelques uns qui prirent la fuite pendant le trajet des colons de Saint-Pétersbourg à Saratov, mais en aucun cas aux colons français de Saratov. Martin n'indique que l'origine de deux familles sur toutes celles qui étaient venues de Saratov à Moscou. La grande divergence des données s'explique sûrement par le décalage qui existait entre les régions qui répondirent par elles-mêmes à l'appel venant de la Russie et celles où l'immigration était engendrée par les recruteurs.

34Sur la base de l'analyse des listes publiées dans Sankt-Peterburgskie vedomosti, 1793, n 47-51.

35 Sur la base de l'analyse statistique du registre consulaire: ADN, Saint-Pétersbourg, consulat, état-civil, $n^{\circ} 1$, Registre des naissances, mariages et décès. An X à 1821 . 
Les Français de Moscou de la deuxième moitié du XIX ${ }^{\mathrm{e}}$ siècle, penchés sur l'histoire de leur communauté, se posaient aussi la question des origines de celle-ci. Leurs opinions à ce sujet sont d'autant plus intéressantes qu'elles pourraient être considérées dans une certaine mesure en tant que jugement de la colonie sur sa propre histoire. Pour Félix Tastevin et Augustin Ladrague, hommes de lettres moscovites français de la fin du XIX ${ }^{\mathrm{e}}$ siècle, la communauté se composait dans un premier temps d'émigrés d'Alsace et de Lorraine. Dans un deuxième temps, des FrancComtois vinrent s'installer à Saint-Pétersbourg et à Moscou après le mariage du grand-duc Paul avec une princesse de Wurtemberg élevée à Montbéliard. Et la troisième vague se situe alors à l'époque de la Révolution et de la Terreur, elle est constituée essentiellement des prêtres et des nobles dont la provenance n'est pas précisée par ces auteurs. A ce flux s'ajoutent quelques petits confluents: les émigrés de la province de Liège et de la Suisse francophone.36 Cette unanimité entre les deux hommes devrait être interprétée comme l'expression des représentations collectives que partageaient les membres de la colonie de cette époque. Ces représentations, sans fausser tout à fait la réalité des choses (telle qu'elle se présente d'après les documents consulaires), ne sont pour autant exemptes de quelques inexactitudes qui sont dues probablement à l'effet du temps et de l'oubli. Cet oubli semble d'autant plus naturel que la colonie se rajeunit considérablement après la dure épreuve subie en 1812 et les nouveaux éléments ne surent pas garder le souvenir exact des débuts de la communauté. En effet, on est frappé par l'oubli absolu du mouvement migratoire de Lyon et du Dauphiné dont les originaires constituaient pourtant plus de $20 \%$ des effectifs de la communauté aux années 1770 (mais dont le nombre diminua rapidement ce qui explique peut-être cet oubli). Le nombre de Parisiens ne semble pas non plus si négligeable, mais est également ignoré par les auteurs cités. Ignorant les sources ou n'y ayant pas accès à l'époque, Tastevin et Ladrague pouvaient prendre pour argent comptant les souvenirs gardés par la mémoire collective de la communauté, imparfaite comme la mémoire de l'homme en général. Il est intéressant à ce propos que l'immigration dont le souvenir persiste surtout dans la communauté, celle des Alsaciens et des Lorrains, paraît une des plus stables et, pour ce qui est des Alsaciens, une des plus récentes.

\section{METIERS DE LA COLONIE. LIEUX DE RESIDENCE.}

RELATIONS AVEC LA NOBLESSE DE MOSCOU.

\section{ORGANISATION STRUCTURELLE DE LA COMMUNAUTE.}

Les précepteurs ne figurent pas dans la liste de 1777 car ils ne formaient pas un corps de métier. Pourtant, cette «profession libérale » de l'époque était indéniablement des plus répandues parmi les Français de Moscou.37 Hormis les précepteurs, le groupe le plus nombreux est constitué par les commerçants. Moins nombreux sont médecins, cuisiniers, perruquiers (le nombre de ces derniers est pourtant important, et ils constituent à Moscou, à la fin du XVIII siècle, une profession spécifiquement française). Plusieurs métiers ne sont représentés que par un ou deux immigrés, comme ceux de chapelier, ferblanquier, horloger, maître d'armes etc.38 A titre de comparaison on peut indiquer les chiffres pour Saint-Pétersbourg.39 Sur environ 800 personnes

36Félix Tastevin, Histoire de la colonie française de Moscou depuis les origines jusqu'à 1812, Paris-M., 1908, p. 34; Ysarn de Villefort, Relation du séjour des Français à Moscou et de l'incendie de cette ville en 1812, par un habitant de Moscou, suivi de divers documents relatifs à cet événement, le tout annoté et publié par A.Gadaruel (Ladrague), Bruxelles, 1871, p. 74-75.

37V. ci-dessous les chiffres pour Saint-Pétersbourg de la fin du XVIII ${ }^{\mathrm{e}}$ siècle qui confirment cette supposition.

38Dans la majorité des cas le métier n'est pas indiqué, ce qui rend presque impossible l'analyse statistique.

39Sur la base des listes du journal Sankt-Peterburgskie vedomosti, 1793, n 45-46. 
adultes sujets français se trouvant à Saint-Pétersbourg en 1793, 85 sont nommés « utchiteli » (professeurs) et 12 gouvernantes (environ 12\% de tous les adultes), 81 sont marchands (10\%), 71 sont militaires (9,3\%), 55 sont dits serviteurs («slugui »»-6,8\%), mais il faudrait y ajouter des valets de chambre («kamerdinery», 2,75\%, ce qui donne en tout presque 10\%), 33 sont cuisiniers (environ 4\%). Parmi les professions moins fréquentes à Saint-Pétersbourg citons les perruquiers (un peu plus de $3 \%$ ) et les artistes $(2,5 \%)$.

Si le préceptorat est un métier très répandu chez les Français Moscovites, comme chez les «Français russes » 40 en général, le commerce est sans doute le métier le plus ancien. Bien que le $3^{\mathrm{e}}$ recensement (1762) n'enregistre pas de marchands français dans les guildes marchandes à Moscou, e les Français commerçaient déjà à Moscou bien avant le début de l'immigration sous Catherine II. Il y en a déjà plusieurs l'année de l'ouverture du vice-consulat de France à Moscou (1759). Ils font des affaires avec Saint-Pétersbourg et avec la France. Un nommé Joseph Gapand vient à Moscou en 1760 avec une provision de tabatières que lui avait confiée un marchand parisien. Mais ayant payé de fortes sommes à la douane, il perd beaucoup d'argent sur cette opération.41 Les Français créent les premières associations, par exemple, pour la conservation de leurs marchandises dans les entrepôts communs. Un tel entrepôt appartenant entre autres aux frères-commerçants Le Maignan et à François Lacroix a brûlé en 1764, ce qui a occasionné une perte de leurs marchandises pour 3109 roubles.42 François La Croix est d'ailleurs un marchand de Saint-Pétersbourg, mais il passe des mois entiers à Moscou pour ses affaires de commerces. Quinze ans plus tard (1777), la deuxième liste connue de Pierre Martin comporte les noms d'environ 3043 marchands français qui sont domiciliés à Moscou. Le $4^{\mathrm{e}}$ recensement (1782) fournit les noms de quatre autres marchands français inscrits dans les guildes qui étaient manifestement à Moscou déjà en 1777 mais sont absents de la liste de Pierre Martin. Sur vingtcinq marchands dont l'origine est connue quatre sont d'anciens Parisiens, trois viennent de Lorraine, un du Dauphiné, une famille de plusieurs personnes est originaire de Provence, une autre vient de Bretagne, une personne de Normandie, de Nîmes, de Grenoble, d'Orange et de Lyon. La spécialisation de quelques uns est indiquée: M.Martin est marchand-bijoutier, M.Servier et M.Chevis vendent des perruques, Mlle Hannevard est marchande de modes. Si en 1782 le nombre des marchands des guildes d'origine française se limite à 7 personnes, ils sont 42 douze ans plus tard, lors du recensement de 1794-1795.44 On rencontre très souvent des noms à consonance française parmi ceux des marchands condamnés à la fin du XVIII siècle à Moscou à de fortes amendes pour la contrebande de marchandises interdites ou importées sans paiement des taxes. 45

La question qui reste à éclaircir concerne les relations entre la population de Moscou (la noblesse mise à part) et les communautés étrangères de cette ville. Il semble qu'avant le début du XIX siècle ces relations dans le milieu marchand n'étaient pas trop tendues. Pourtant le nombre des libraires français toujours croissant à Moscou ne pouvait, semble-t-il, que susciter le mécontentement de leurs confrères russes. S'il n'en était point ainsi pendant longtemps, c'est peut-être dû à leurs différences: les lieux d'activité privilégiés par les premiers (le Pont des

40Lancé par les soldats de la Grande Armée aux Français Moscovites, ce mot, à y bien penser, a quelque chose de juste, tout en restant un paradoxe.

41ADN, Moscou, consulat, $\mathrm{n}^{\circ} 1$, p. 54.

42Ibid., p. 167-168.

43On ne sait pas combien étaient les frères Le Maignan ou les frères de Landre.

44Najdenov, op.cit., vol. 4, p. 434-474.

45История Московского купеческого общества: сословно-общественная деятельность Московского купечества в XIX веке, vol. 2, t. 1, p. 40. Ce fait donnera un excellent prétexte pour une campagne initiée par les marchands russes de Moscou contre les étrangers en 1805. 
maréchaux, Tverskaïa, Rojdestvenka etc.) se trouvaient à l'écart du Pont du Saint-Sauveur où vendaient les livres les libraires russes de Moscou, ce qui s'explique par la différence de la clientèle qu'ils servaient. Les Français se spécialisèrent dans le premier temps dans les livres étrangers et n'empiétaient pas tellement sur l'activité des libraires russes. Certains domaines du commerce français étaient sans doute à l'abri de la jalousie des marchands russes comme étant très spécifiques (le commerce des perruques, de certains articles de mode, etc.). En outre, beaucoup de marchands russes de Moscou étaient eux-mêmes des «intrus », venus d'autres villes de l'Empire.

Si à la fin du XVIII ${ }^{\mathrm{e}}$ siècle non moins de $30 \%$ des immigrés français à Saint-Pétersbourg dépendaient directement de l'aristocratie, essentiellement russe, qu'ils servaient,46 la proportion des Français au service de la noblesse était probablement encore plus importante à Moscou.47 On pourrait dire pour cette raison que les besoins de l'aristocratie déterminaient dans une grande mesure la constitution de la colonie du point de vue professionnelle et notamment le nombre de ceux qui exerçaient tel ou tel métier, quoiqu'on ne doive pas oublier que, en retour, les Français exerçaient une influence indéniable sur la formation de ces besoins. La demande des précepteurs saturée dans la nouvelle capitale, le trop-plein de ceux-ci se déversait sur Moscou. A la fin du $\mathrm{XVIII}^{\mathrm{e}}$ - début du $\mathrm{XIX}^{\mathrm{e}}$ siècle apparaissent dans le journal officiel de Moscou Moskovskie vedomosti des annonces de Français proposant leurs services en tant que précepteurs à la noblesse provinciale. Serait-il devenu si difficile de trouver une place à Moscou vers cette époque?

Avec quelle couche de la population de Moscou les Français-Moscovites entretenaient-ils de préférence les relations? Par qui étaient-ils le plus souvent engagés? Qui achetait les livres dans les librairies fondées par les Français? Il est vrai qu'en répondant que la noblesse de Moscou était un interlocuteur social privilégié de la communauté francophone à Moscou, on ne risque pas de se tromper, mais on reste pourtant sur sa faim. On ne peut pas encore donner une réponse détaillée à chacune des questions posées, mais les documents accessibles permettent néanmoins de fournir des éléments de réponse.

Un Français Emmanuel Bousson de Mairet se trouve au service du comte Nikita Petrovic Panin, futur vice-chancelier. C'est un des rares Français récalcitrants qui refuse de prêter le serment en 1793 et est obligé de quitter l'Empire de Russie. Dans les années 1770, on trouve dans la famille du général-en-chef de l'armée russe, comte Valentin Platonovitch Moussine-Pouchkine, un ancien officier français Nicolas Henri Feriel du Foy, en qualité de gouverneur. Dans les années 1780 un nommé Jacques Chevenet est chef de cuisine du brigadier comte Stepan Stepanovitch Apraksine. Son homologue Jean Baptiste Maiz sert le feld-maréchal comte Ivan Petrovitch Saltykov, gouverneur de Moscou depuis 1797.48 Dans les listes des parrains des enfants des

46On a en vue les précepteurs, les cuisiniers, les maîtres d'hôtel, etc. qui étaient effectivement directement dépendants des nobles qui les engageaient, à la différence, disons, des marchands ou des militaires.

47Tandis que les militaires faisaient presque défaut dans la communauté française de Moscou à la fin du XVIII ${ }^{\mathrm{e}}$ siècle.

48J'exprime d'autant plus ma reconnaissance à Vladimir Somov de m'avoir communiqué ces informations qu'elles sont contenues dans les dossiers conservés aux Archives diplomatiques de Nantes mais faisant partie du fonds non répertorié et actuellement inaccessible aux chercheurs. On ne dispose jusqu'à présent ni en France ni en Russie d'aucune étude sérieuse sur les commuautés françaises en Russie qui reposât sur des données aussi concrètes. Dans cette situation, on ne peut que donner raison à Vl. Somov lorsqu'il soulignait combien importantes seraient des recherches dans le fonds des archives des anciens postes diplomatiques français en Russie conservé aux Archives diplomatiques de Nantes. A grand regret, la majeure partie de ce fonds attend d'être reclassée et reste fermée aux historiens. Cf. Vl. Somov, Материаль по истории русско-французских связей XVIII века из Архива франиузского посольства в Санкт-Петербурге (Нант) // Петербургские чтения, résumés des communicatons du colloque qui s'est tenu à Saint-Pétersbourg en 1994, p. 158-160. V. aussi l'article de Vl. Somov, V. Rjéoutski, Франиузы в России в эпоху Просвещения (материальк к истории русско-франиузских связей 1760-1780х-г2. 
immigrés français baptisés à l'église catholique des Saints-Apôtres-Pierre-et-Paul à Moscou en 1783 surgissent les noms de Fiodor Pavlovitch Balk-Polev, chambellan actuel; de la comtesse Ekaterina Ivanovna Chouvalov, veuve du feld-maréchal; du conseiller privé actuel grand chambellan et sénateur prince Alexandre Mikhaïlovitch Golitsyne. La même année d'autres aristocrates russes de Moscou parrainent des enfants français dans la même église: le comte Piotr Alexandrovitch Boutourline, conseiller privé, fils du feld-maréchal, la comtesse Ekaterina Ivanovna Golovkina, épouse du comte Mikhaïl Gavrilovitch Golovkine, vice-chancellier et sénateur exilé sous l'impératrice Elisabeth. Cette énumération des grands noms titrés de l'Empire pourrait être prolongée presque à l'infini: les noms des princes Troubetskoï, Khavanski, Odoïevski, Gagarine, Dolgorouki, Volkonski, des comtes Cheremetev, Apraksine etc. se mêlent aux noms des étrangers obscurs.49 Cette longue liste témoigne des relations assez étroites établies vers cette époque entre la haute noblesse de Moscou et les étrangers. Elle montre aussi, bien qu'indirectement, que la communauté francophone de Moscou est entrée dans la phase de l'épanouissement et de la maturité dans la deuxième partie du règne de Catherine II.

Qui plus est, on pourrait dire que le rapprochement avec la noblesse de Moscou se produit aussi dans le sens propre du terme. Selon les usages du temps les précepteurs, les cuisiniers, les maîtres d'hôtel, etc. vivaient d'habitude dans les maisons de la noblesse qui les engageait. De ce fait, ils entraient en contact avec l'aristocratie journellement et souvent, si on pense aux précepteurs et secrétaires, de façon presque intime. D'autre part, ces Français étaient, du point de vue du lieu de leur résidence, exclus, dans une certaine mesure, de la vie de la communauté. C'est probablement une des explications du fait que les Français, étant déjà présents et fort nombreux à Moscou au début des années 1760, étaient quasiment introuvables dans le faubourg des Etrangers. Les listes des recensements concernant les marchands des guildes de Moscou50 indiquent le «trajet » qu'avait effectué la colonie francophone à Moscou depuis les années 1760 à la fin du XVIII ${ }^{\mathrm{e}}$ siècle. Lors du quatrième recensement (1782), 7 marchands français inscrits dans les guildes marchandes habitent différents quartiers de Moscou, y compris 2 qui résident dans le faubourg des Etrangers, mais on n'en trouve aucun à cette époque dans le quartier de Loubianka et de la rue du Pont des maréchaux (Kouznetski most). Douze ans plus tard, lors du cinquième recensement (1794-1795), sur 22 marchands français inscrits dans les guildes dont les adresses sont indiquées, 16 habitent dans le quartier de Loubianka et 10 de ce nombre directement «près de la rue du Pont des maréchaux» ou «sur le Pont des maréchaux ». Ce fait mérite explication. On est enclin d'en voir les mobiles essentiellement dans le changement des circonstances qui a, en l'espace de dix ou quinze ans, valorisé ce quartier, surtout au regard des communautés étrangères, qui étaient en grande partie au service de l'aristocratie et s'orientaient sur ses goûts. Un des comtes Vorontsov a construit, dès le milieu du siècle, plusieurs maisons prestigieuses, sur la Rojdestvenka et le long de la rivière Neglinka. Beaucoup d'autres aristocrates l'ont suivi, transformant ainsi cette espace en un quartier cossu. Sous Catherine II, il fut permis de commercer partout dans la ville, et non seulement dans l'enceinte des Galeries marchandes comme jusque là.

Les Français habitaient les uns près des autres. Ainsi, dans la maison du général Beketov, près de la rue du Pont des maréchaux, étaient logés les marchands français Magdeleine Renière, Jacques

из архива франиузского посольства в Санкт-Петербурге) // Западно-европейская культура в книгах и рукописях Российской национальной библиотеке. Памяти А. Д. и В. С. Люблинских. СПб., изд. Дмитрий Буланин, 2001, р. 285-299.

49Documents des églises catholiques de Moscou, BNR, département des manuscrits, raznojaz., F-II, $\mathrm{n}^{\circ}$ 27/1, f. 81 $s q q$. On peut penser qu'il s'agit des seigneurs de ces mêmes Français dont les enfants étaient parrainés par ces aristocrates. Or ce n'est pas le cas, sinon il faudrait admettre que ces Français pouvaient passer tout le temps d'un seigneur à l'autre.

50Najdenov, op.cit., vol.3-4. 
Gay, libraire, Robert Philippeau avec son épouse Louise Villaume. Dans la maison du libraire François Courtener logeaient aussi quelques Français, notamment Jean Gautier, futur beau-fils de Courtener, et François Riss, aussi libraire.51

Les Français firent de grands progrès dans la vente des livres à Moscou, montrant ainsi aux libraires russes les horizons dans l'art de librairie. De Marey, les frères Gay, Courtener, Bugnet, Gautier, Riss et Saucet introduisirent les catalogues, les salles de lecture, le prêt des livres tant aux Moscovites qu'aux provinciaux, la publicité des nouveautés de librairie dans les journaux de Moscou, etc. Un développement extraordinaire des librairies françaises tenues par des Français était dû naturellement au contexte culturel et social de l'époque, mais peut-être aussi à un fait curieux: on a pu constater que plusieurs libraires français de Moscou étaient originaires de la même ville, Strasbourg, ils se connaissaient sans doute avant le départ en Russie, peut-être leur départ même était-il coordonné.52 Il est remarquable que les frères Gay par exemple, commencent à vendre des livres d'abord à Moscou, puis élargissent leur commerce en ouvrant en 1787 leur magasin (qui existera jusqu'en 1800) à Saint-Pétersbourg en pleine perspective Nevski.53 D'autres Français, tel François Courtener, ayant de proches parents libraires à l'étranger, exploitent habilement la possibilité de commander des nouveautés par leur intermédiaire. Ainsi, Courtener fait paraître une Note de livres nouveaux et autres, en vente chez Pierre Courtener, libraire à Basle, et chez François Courtener, libraire à Moscou.54 Les catalogues édités par la maison Courtener permettent de suivre l'évolution de son commerce. Ayant ouvert d'abord aux années 1780 une librairie «rue Illienski à Moscou aux Boutiques Neuves de Mr. Calinin, $n^{\circ} 3$ », c'est-à-dire dans la rue Iliinski, Courtener ouvre bientôt un autre magasin de livres, cette fois «rue Nikolsky, vis-à-vis de Zaïkonospasky monastère », «maison de Schewaltischeff ». Son Catalogue methodique comptait 150 pages.55

On est amené, sur la base des documents quelque peu modestes dont nous disposons, à supposer que les marchands français tendaient à conclure à cette époque les mariages le plus souvent dans leur propre milieu, celui des marchands français. Jean-Marie Gautier Dufayer qui vient en Russie à la fin des années 1750 avec quelques compagnons, change en quelques années plusieurs professions, y compris celle - incontournable -, de précepteur, avant de passer de SaintPétersbourg à Moscou et de s'inscrire comme marchand des guildes avec le capital d'un millier de roubles qu'il arrive à réunir sur les économies de son salaire de gouverneur, de directeur d'une manufacture privée, etc. Bientôt, marchand de la première guilde à Moscou, il épouse Jeanne Gaudain, fille du directeur du Club Anglais à Moscou, lui aussi Français. Jean Gautier, alias Ivan Ivanovitch Gautier, issu de cette union, s'apparente au libraire François Courtener par mariage avec sa fille et il héritera la maison Courtener qui durera encore un siècle.

Dans les années 1780-1790 les unions «intercommunautaires » ou «interethniques » ne sont plus si rares.56 Ainsi, Mlle Rose Hannevard, soeur des frères Hannevard qui ont à cette époque à

51Ibid., vol. 4; Martynova-Poniatovskaïa, op.cit.

52V. au sujet des libraires français de la fin du XVIII ${ }^{\mathrm{e}}$ - début du XIX ${ }^{\mathrm{e}}$ siècle à Moscou les articles cités de Martynova-Poniatovskaïa, op.cit., vol. 1, Начало франиузской книжной торговли в Москве, р. 113-131, Книжная торговля Готье (1799-1812) и Дандильи (1805-1810), p. 153-180. Faits essentiellement sur la base d'une seule source, le journal Moskovskie vedomosti, ces articles fournissent une information d'une richesse exceptionnelle.

53Сводный каталог книг на иностранных языках, изданных в России в XVIII веке. L., 1985, vol. 3, p. 180.

54Se trouve au RGADA ; cf. ibid., vol.3, p.191.

55Ibid., vol. 3, p. 192.

56Cette conclusion découle de l'analyse des registres de mariages et de baptêmes des églises catholiques de Moscou: églises des Saints Apôtres Pierre et Paul et Saint Louis des Français. Bibliothèque nationale de Russie, département des manuscrits, raznojaz., F-II, n 27/1, f. 81-109, n 27/2, f. 552 et suiv. 
Moscou une importante maison de commerce, se marie avec le fils du propriétaire d'une fabrique de tapisseries, l'Anglais Martin Butler. Cette union est probablement d'autant plus avantageuse pour les Hannevard qu'elle coïncide avec la période de grandes difficultés financières qu'éprouve la maison «Hannevard, Becker et Cie». En 1775, les marchands sont obligés de présenter au consulat de France à Saint-Pétersbourg le bilan de leur commerce pour que la chancellerie puisse procéder à la liquidation de la société.57

Il semble que jusqu'à la fin du XVIII ${ }^{\mathrm{e}}$ siècle les mariages des Français avec des Russes n'étaient somme toute pas fréquents. Si dans la liste de 1793 publiée dans le journal Moskovskie vedomosti les noms de jeune fille des femmes des Français sont presque toujours mentionnés, il n'y a pas parmi elles aucune qui soit Russe.58 C'est d'ailleurs naturel puisqu'il s'agit d'une liste des sujets français. Les noms à consonance russe sont tout autant absents des registres des mariages de l'église Saint-Louis des Français: de 1790, quand le registre fut ouvert, à l'année 1793 incluse, il n'y en a pas un seul.59 Cela ne signifie pas que les mariages «mixtes » entre les Russes et les Français étaient inexistants, mais il faut en chercher les traces ailleurs. A titre d'exemple, citons une des plus nombreuses familles françaises de Moscou, les Dellesalle. Sur le nombre présumé de treize enfants qu'avait le fondateur de cette famille Philippe Dellesalle, nés dans les années 1760-1770, quatre épousent leurs compatriotes établis à Moscou; trois autres se marient à des représentants d'autres communautés étrangères de Moscou, sans doute des Allemands à juger par leurs noms; trois autres épousent des Russes, dont Piotr Iakovlevitch Anitchkov, assesseur de collège et propriétaire à Moscou; les noms des conjoints des trois qui restent sont inconnus pour le moment. Il s'agit d'une famille qui visait plutôt la plus rapide assimilation, donc n'aurait pas dû se cantonner dans les limites de la rue du Pont des maréchaux. Une branche des Dellesalle rompt même ses attaches avec la communauté française et s'installe à Saint-Pétersbourg où l'attendait une importante ascension sociale. La grande réussite sociale, dans une carrière militaire ou civile, qui donnerait l'accès à la noblesse, supposait pour une famille française à Moscou, de relacher les liens avec la communauté et d'accepter la plus rapide assimilation linguistique et culturelle.60 Les contraintes des circonstances jouaient aussi leur rôle dans les stratégies maritales. Pour une Française de Moscou, épouser un Français n'est pas toujours facile car le choix des conjoints français n’est pas illimité ; les Français sont amenés parfois à enfreindre certaines règles de bienséance généralement reconnues à l'époque. Ainsi, la fille de Sophie Philippovna Dellesalle et de Pierre Dunoyer épouse Alexandre Gavrilovitch Dellesalle qui était son cousin germain.61

Une des fonctions de l'église Saint Louis des Français à Moscou était justement de mettre une barrière ou du moins de ralentir le processus de brassage de la population française de Moscou avec les autochtones et d'autres étrangers. L'initiative de fonder une église « française » vint de plusieurs familles respectables de la colonie, du vice-consul et, naturellement, des ecclésiastiques

$\underline{57 R e ́ c a p i t u l a t i o n ~ o u ~ E t a t ~ d e ~ t o u s ~ l e s ~ a c t e s ~ p a s s e ́ s ~ e t ~ c o n t e n u s ~ d a n s ~ l e ~ R e g i s t r e ~ d u ~ C o n s u l a t ~ G e ́ n e ́ r a l ~ d e ~ F r a n c e ~ a ̀ ~}$ Saint-Pétersbourg, AN, Affaires étrangères, B-I-988, f. 138-139.

58Moskovskie vedomosti, $1793, \mathrm{n}^{\circ} 47-51$.

59Bibliothèque nationaloe de Russie, département des Manuscrits, raznojaz., F-II, n 27/1, loc. cit.

60L'apparition d'une importante couche de la «nouvelle noblesse », de la noblesse de service pour la plus grande partie, parmi les Français de Moscou dans la première moitié du XIX ${ }^{\mathrm{e}}$ siècle et l'importance de ce phénomène pour la compréhension des processus d'assimilation et de la résistance à l'assimilation, devront encore être étudiés.

61La généalogie de cette famille est reconstituée d'après les sources multiples, notamment inédites: le fonds des églises catholiques de Moscou, BNR, département des Manuscrits, raznojaz., F-II, n 27/1; Archives historiques d'Etat de Russie (RGIA), F. 1343, inv. 3, dossier 663; inv. 20, dossiers 936-938. 
français qui étaient des hôtes de plus en plus fréquents à Moscou et s'y établissaient même.62 En août 1789 tous les Français de la communauté reçurent une invitation à venir participer à une réunion générale de la colonie. Le texte de cet imprimé annonçait :

«Monsieur! [suit le nom de celui à qui la feuille était adressée] Comme membre de la Communauté françoise, vous êtes prié de vous trouver dimanche prochain 5 août à 9 heures du matin à l'Assemblée qui se tiendra chez M. de Bosse, Sindic,63 à l'Oustretinka,64 pour régler la communauté Catholique françoise, séparée des autres par la permission de Mgr. l'Archéveque de Mohilew; d'aviser aux moyens d'établir ses revenues, ses casuels etc. et de pourvoir à ses frais; réunion qui doit flâter les françois jaloux de conserver l'intimité nationale et les ressources qu'elle offrira dans toutes les positions malheureuses; réunissant aussi l 'agrément d'avoir son prêtre, ses registres; de suivre ses usages pour le service divin et d'éviter à l'avenir les contestations qui naissent naturellement de la différence du génie de 4 nations melées ensemble ».65 L'assemblée de la communauté résolut de «former une communauté particulière et d'élever une église ou le culte catholique put se célébrer selon les rites de sa nation, en vertu des traités entre la France et la Russie ».66

La communauté nomma plusieurs syndics et leurs adjoints. Et c'est le 30 mars 1791 que l'église fut bénie et dédiée à Saint Louis, Roi de France, « en présence de la communauté généralement rassemblée et avec un concours égal de la noblesse russe ».67 En 1792, peut-être à la demande des prêtres français, l'archevêque prescrivit la délimitation complète de la paroisse française et enjoignit aux desservants de l'église de ne confesser et communier que leurs ouailles. On en vint à interdire à un Italien, Gambotti, d'occuper le poste de syndic de la communauté, bien que l'intéressé fût de plein gré accepté dans la colonie, étant probablement parfaitement francophone. 68

\section{CONCLUSIONS}

En tirant quelques conclusions préalables, soulignons une fois de plus que ce fut sous le règne de Catherine II que la communauté française (ou, plus justement, francophone), d'une petite poignée, se transforma en une colonie de dimensions considérables et cela, en grande partie grâce au mouvement de population provoqué par la politique migratoire de Catherine II, bien qu'à l'encontre des ojectifs que visait le gouvernement. Si pourtant dans une ville comme Moscou, le «poids » de cette communauté était des plus modestes, elle ne jouait pas moins un rôle important dans le fonctionnement de la ville et dans la conscience publique.69 Ceci était dû d'abord au fait que la communauté francophone de Moscou réussit à établir des relations avec de très larges couches de l'ancienne capitale et surtout avec l'aristocratie moscovite; et puis grâce à

62Comme le fera l'abbé Adrian Surugue, pendant plusieurs années, après avoir quitté la pension de l'abbé Nicole à Saint-Pétersbourg, engagé en tant que précepteur dans la famille du comte A.Musin-Puškin, président du SaintSynode, découvreur du Dit de l'Ost d'Igor.

63Mais d'abord et surtout vice-consul de France qui remplaça Pierre Martin.

64Il s'agit naturellement de Sretenka.

65Documents des églises catholiques de Moscou, BNR, département des Manuscrits, raznojaz., F-II, n 27/1, f. 291.

66Documents officiels et procès-verbaux ayant rapport aux fondations diverses des Français domiciliés à Moscou, 1789-1892, Moscou, 1892, p. 14-17.

67Ibid.

68Ibid., p.21.

69Dès la fin du XIX ${ }^{\mathrm{e}}$ siècle le «Pont des Maréchaux » devient le synonyme de la présence des Français à Moscou, de la communauté française et de l'influence française. 
l'organisation de la communauté autour du vice-consulat et de l'église catholique française de Moscou. C'est cette dernière particularité qui permettra à la communauté de se maintenir encore très longtemps en tant que groupe « ethnique ». 